increasing interest in administration by continuous infusion in adult studies, but paediatric data is lacking. A continuous infusion, if given via an elastomeric pump could also facilitate earlier discharge from hospital.

Methods Children with an oncology/haematology diagnosis admitted to Alder Hey Children's Hospital with febrile neutropenia, normally treated with Piperacillin/Tazobactam via elastomeric device were considered eligible for the study. Patients received 24-36 hours of intermittent dosing before continuous dosing commenced via elastomeric pump. We analysed the data from 5 patients with double lumen central lines as a pilot phase to determine if expanded recruitment to include patients with single lumen lines was possible.

Results Five patients were recruited, four of which had the continuous infusion. The mean Cmax following intermittent dosing of piperacillin and tazobactam from the lumen used for drug administration were $189.7 \mathrm{mg} / \mathrm{L}, 95 \%$ CI $[71.7$ 307.9] and $18.5 \mathrm{mg} / \mathrm{L}, 95 \%$ CI [10.7, 26.3] respectively. The mean Cmax following intermittent dosing for the 'empty' lumen were $160.5 \mathrm{mg} / \mathrm{L}$, 95\% CI [121.6 - 199.4] for piperacillin and $13.7 \mathrm{mg} / \mathrm{L}, 95 \%$ CI [11.2 - 16.3] for tazobactam. The largest difference seen was on patient 002 with a concentration of piperacillin almost double that seen in the 'empty' lumen (429 mg/L vs $233 \mathrm{mg} / \mathrm{L})$.

Conclusion The lumen used for drug administration has enough residual drug to influence the results, so expanding recruitment to include single lumen lines is not going to be undertaken. The study has been amended to recognise this, and recruitment will continue with double lumen central lines only.

Disclosure(s) Nothing to disclose

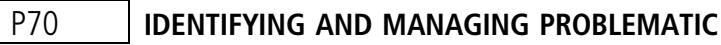 POLYPHARMACY IN CHILDREN AND YOUNG PEOPLE?}

${ }^{1,2}$ J Moss*, ${ }^{3} \mathrm{R}$ Austin, ${ }^{3,4} \mathrm{D}$ Hawcutt. ${ }^{1}$ Paediatric Medicines Research Unit, Alder Hey Children's NHS Foundation Trust; ${ }^{2}$ Department of Women's and Children's Health, Institute of Translational Medicine; ${ }^{3} 2$ Department of Women's and Children's Health, Institute of Translational Medicine, University of Liverpool; ${ }^{4}$ NIHR Alder Hey Clinical Research Facility, Alder Hey Children's NHS Foundation Trust, Liverpool, UK

\subsection{6/archdischild-2019-esdppp.108}

Background Polypharmacy may be necessary in a patient with complex disease or multiple illnesses. Problematic polypharmacy (PP) is defined as the prescribing of multiple medications inappropriately, or where the intended benefit of the medication is not realised. Identification and management of PP already occurs in adult medicine, with evidence based guidelines and deprescribing tools available. This work aims to establish the extent of polypharmacy affecting children at our centre, and the existence of evidence based de-prescribing guidelines to manage potential PP.

Methods Audit of children and young people prescribed medications at a secondary and tertiary children's hospital, January - December 2017. A systematic review looking for evidence of such a deprescribing tool or guideline was registered with PROSPERO, and undertaken. Inclusion criteria specified the need for a guideline or deprescribing tool in children from birth to $<18$ yrs of age. Two independent reviewers performed the review with a third reviewer resolving any discrepancies.

Results Within a secondary and tertiary care children's hospital, 668 children were identified as receiving $\geq 10$ drugs concurrently, while 30 were receiving $\geq 20$ drugs at one time.

The systematic review identified 563 papers initially, with 482 remaining once duplicates were removed. After application of inclusion and exclusion criteria, two studies were included, POPI (Prot-Labarthe et al, 2014) and PIPc (Barry et al, 2016). These papers discuss systems to identify inappropriate prescriptions. No evidence based guidelines related to the management of potential PP in children were identified.

Conclusion There are children receiving a large number of medicines concurrently, putting them at risk of PP. No specific deprescribing guideline or tools to guide management were identified. Paediatric clinical pharmacology is well placed to create and implement such guidelines.

Disclosure(s) Nothing to disclose

\section{P71 POPULATION PHARMACOKINETICS AND PHARMACOGENETICS OF CAFFEINE IN CHINESE PREMATURE INFANTS WITH APNOEA OF PREMATURITY}

${ }^{1} \mathrm{~S} \mathrm{Ni}{ }^{*},{ }^{2} \mathrm{X}-\mathrm{B} \mathrm{Gao},{ }^{3} \mathrm{Hu} \mathrm{Y}-\mathrm{E},{ }^{2} \mathrm{C}-\mathrm{H}$ Wang, ${ }^{2} \mathrm{Z}-\mathrm{H}$ Jiang, ${ }^{3} \mathrm{~F}$ Yang, ${ }^{3} \mathrm{Y}$ Zheng, ${ }^{4} \mathrm{~W}$ Zhao. ${ }^{1}$ The National Clinical Trial Institute; ${ }^{2}$ Children's Hospital, Zhejiang University School of Medicine, hangzhou; ${ }^{3}$ School of Pharmaceutical Sciences, Shandong University; ${ }^{4}$ Shandong Provincial Qianfoshan Hyspital, Shandong University, Jinan, China

10.1136/archdischild-2019-esdppp.109

Background Caffeine is commonly regarded as the treatment of choice for neonatal apnoea. However, limited data on the developmental pharmacokinetics and pharmacogenetics were available in Chinese premature infants. The objective of this study was to develop a population pharmacokinetic model of caffeine after intravenous administration to Chinese neonates with apnoea of prematurity (AOP) and evaluate the impact of developmental pharmacogenetics of CYP1A2.

Methods Sparse pharmacokinetic samples were collected from AOP newborns receiving caffeine citrate at a loading dose of $20 \mathrm{mg} / \mathrm{kg} / \mathrm{d}$ and maintenance dose of $5-10 \mathrm{mg} / \mathrm{kg} / \mathrm{d}$. Population pharmacokinetic-pharmacogenetic analysis of caffeine was performed using NONMEM. Eight CYP1A2 polymorphisms were genotyped.

Results A total of 99 newborns with a mean (SD) postmenstrual age of 32.0 (2.16) (range 22.3 - 38.0) weeks were included in the present study. Pharmacokinetic data fitted an one-compartment model with first-order absorption and elimination. Current weight, postmenstrual age and serum creatinine concentration were significant covariates influencing caffeine clearance. None of tested CYP1A2 polymorphisms had significant impact on caffeine pharmacokinetics.

Conclusion The population pharmacokinetics-pharmacogenetics of caffeine was evaluated in Chinese AOP premature infants. This developmental pharmacokinetic model can be helpful to individualize caffeine therapy.

Disclosure(s) Nothing to disclose 